WATPHYS-TH01/02

\title{
Quasilocal Thermodynamics of Kerr and Kerr-anti-de Sitter Spacetimes and the AdS/CFT Correspondence
}

\author{
M.H. Dehghani円 and R. B. Mann[ \\ Department of Physics, University of Waterloo, \\ Waterloo, Ontario N2L 3G1, CANADA
}

PACS numbers: 04.60.-m,04.70.-s,04.70.Dy,11.25.Db

October 28, 2018

\begin{abstract}
We consider the quasilocal thermodynamics of rotating black holes in asymptotically flat and asymptotically anti de Sitter (AdS) spacetimes. Using the minimal number of intrinsic boundary counterterms inspired by the AdS/conformal field theory correspondence, we find that we are able to carry out an analysis of the thermodynamics of these black holes for virtually all possible values of the rotation parameter and cosmological constant that leave the quasilocal boundary well defined, going well beyond what is possible with background subtraction methods. Specifically, we compute the quasilocal energy $E$ and angular momentum $J$ for arbitrary values of the rotation, mass and cosmological constant parameters for the $(3+1)$ - dimensional Kerr, Kerr-AdS black holes and $(2+1)$ - dimensional Banados Teittelboim and Zanelli (BTZ) black hole. We perform a quasilocal stability analysis and find phase behavior that is commensurate with previous analyses carried out at infinity.
\end{abstract}

\footnotetext{
${ }^{1}$ Electronic address: hossein@avatar.uwaterloo.ca; On leave from Physics Dept., College of Sciences, Shiraz University, Shiraz, Iran

${ }^{2}$ EMail: mann@avatar.uwaterloo.ca
} 


\section{Introduction}

Asymptotically anti-de Sitter (AdS) spacetimes continue to attract a great deal of attention, primarily because of the recently conjectured AdS conformal field theory (CFT) correspondence principle. This principle posits a relationship between supergravity or string theory in bulk AdS spacetimes and conformal field theories on the boundary. It offers the tantalizing possibility that a full quantum theory of gravity could be described by a well understood CFT-Yang-Mills theory (albeit with the asymptotic AdS criterion). Observables in the quantum gravity theory could be computed using quantum field-theoretic methods, and vice versa. Although there is no proof of the conjecture, a dictionary is emerging that translates between different quantities in the bulk gravity theory and its counterparts on the boundary, including the partition functions and correlation functions of each. A set of quantities of particular interest are those associated with gravitational thermodynamics. It has long been known that a physical entropy $S$ and temperature $\beta^{-1}$ can be ascribed to a given black hole configuration, where these quantities are respectively proportional to the area and surface gravity of the event horizon(s) [1, 2]. Other black hole properties, such as energy, angular momentum and conserved charge can also be given a thermodynamic interpretation [3]. More recently it has been shown that entropy can be associated with a broader and qualitatively different object called a Misner string, which is the gravitational analogue of a Dirac string [田, 5, 6, 7, 8, 9].

In general the problem of defining and calculating gravitational thermodynamic quantities remains a lively subject of interest. Because they typically diverge for both asymptotically flat and asymptotically AdS spacetimes, a common approach toward evaluating them has been to carry out all computations relative to some other spacetime that is regarded as the ground state for the class of spacetimes of interest. This is done by taking the original action $I_{G}=I_{\mathcal{M}}+I_{\partial \mathcal{M}}$ for gravity coupled to matter fields and subtracting from it a reference action $I_{0}$, which is a functional of the induced metric $\gamma$ on the boundary $\partial \mathcal{M}$. Conserved and/or thermodynamic quantities are then computed relative to this boundary, which can then be taken to (spatial) infinity if desired.

This approach has been widely successful in providing a description of gravitational thermodynamics in regions of both finite and infinite spatial extent 10, 11. Unfortunately it suffers from several drawbacks. The choice of reference spacetime is not always unique [12], nor is it always possible to embed a boundary with a given induced metric into the reference background. Indeed, for Kerr spacetimes, this latter problem forms a serious obstruction towards calculating the subtraction energy, and calculations have only been performed in the slow-rotating regime [13].

An extension of this approach, which addresses these difficulties was recently developed based on the conjectured AdS/CFT correspondence [14, 15, 16, 17]. Since quantum field theories in general contain counterterms, it is natural from the AdS/CFT viewpoint to append a boundary term $I_{c t}$ to the action that depends on the intrinsic geometry of the (timelike) boundary at large spatial distances. This requirement, along with general covariance, implies that these terms be functionals of curvature invariants of the induced metric and have no dependence on the extrinsic curvature of the boundary. An algorithmic procedure [18] exists for constructing $I_{c t}$ for asymptotically AdS spacetimes, and so its determination is unique. The addition of $I_{c t}$ will not affect the bulk equations of motion, thereby eliminating the need to embed the given geometry in a reference 
spacetime. Hence thermodynamic and conserved quantities can now be calculated intrinsically for any given spacetime. The efficacy of this approach has been demonstrated in a broad range of examples, all of them in the spatially infinite limit, where the AdS/CFT correspondence applies [5, 6, 8, 19, 20]. However this limit is an idealization, and can never be satisfied in reality.

The purpose of this paper is to investigate the effects of including $I_{c t}$ for quasilocal gravitational thermodynamics for situations in which the region enclosed by $\partial \mathcal{M}$ is spatially finite. There are several reasons for considering this. Although the AdS/CFT correspondence is conjectured to be valid only at infinity, from a gravitational viewpoint the inclusion of additional boundary functionals is not uniquely dependent upon this correspondence and could in fact be carried out even if the conjecture is ultimately found to be invalid. It is, therefore of interest to explore the implications of $I_{c t}$ outside of its original conceptual framework. Furthermore, the inclusion of $I_{c t}$ eliminates the embedding problem from consideration whether or not the spatially infinite limit is taken, and it is of interest to see what its impact is on quasilocal thermodynamics. Since $I_{c t}$ is a functional of the induced metric, it will in general depend upon parameters in the solution related to conserved quantities at infinity, and could conceivably alter the results obtained for quasilocal thermodynamics relative to a fixed background. Of course $I_{c t}$ itself is no longer unique, since one could add any functional of curvature invariants that vanished at infinity in a given dimension. However the expression for $I_{c t}$ obtained by the algorithm given in Ref. [18 is the minimal one required to eliminate divergences, and therefore merits study.

We specifically consider in this paper an investigation of the thermodynamic properties of the class of Kerr and Kerr AdS black holes in the context of spatially finite boundary conditions, with the boundary action supplemented by $I_{c t}$. With their lower degree of symmetry relative to spherically symmetric black holes, these spacetimes allow for a more detailed study of the consequences of including $I_{c t}$ for spatially finite boundaries. Although the inclusion of $I_{c t}$ was originally proposed for asymptotically AdS spacetimes, it has been demonstrated that it can be extended to asymptotically flat spacetimes [5, 8, 26] under certain topological constraints [18]. We consider the implications of this extension for Kerr black holes in spatially finite regions.

The outline of our paper is as follows. We review the basic formalism in Sec. 2. In the next two sections we compute the quasilocal energy, mass, and angular momentum for the Kerr and Kerranti-de Sitter black holes respectively. These quantities must be computed numerically for general values of the parameters, and we present our results graphically. We perform a simple stability analysis for each case, and compare this to previous analyses carried out at infinity [21, 22]. We finish our paper with some concluding remarks.

\section{General Formalism}

The action is the sum of three kinds of terms. The first two are a volume (or bulk) term

$$
I_{v}=-\frac{1}{16 \pi} \int_{\mathcal{M}} d^{4} x \sqrt{-g}[\mathcal{R}-2 \Lambda+\mathcal{L}(\Phi)]
$$

and a boundary term

$$
I_{b}=-\frac{1}{8 \pi} \int_{\partial \mathcal{M}} d^{3} x \sqrt{-\gamma} \Theta(\gamma)
$$


chosen so that the variational principle is well defined. The Euclidean manifold $\mathcal{M}$ has the metric $g_{\mu \nu}$, covariant derivative $\nabla_{\mu}$, and time coordinate $\tau$ which foliates $\mathcal{M}$ into non-singular hypersurfaces $\Sigma_{\tau}$ with unit normal $u_{\mu}$ over a real line interval $\Upsilon$. $\Theta$ is the trace of the extrinsic curvature $\Theta^{\mu \nu}$ of any boundary(ies) $\partial \mathcal{M}$ of the manifold $\mathcal{M}$, with the induced metric(s) $\gamma_{i j}$; the manifold can have internal boundary components as well as a boundary at infinity, although only the latter will be needed in what follows. Here $\mathcal{L}(\Phi)$ is the matter Lagrangian and $\Lambda$ the cosmological constant, which, in what follows, will be taken to be either zero or negative.

In general $I_{v}$ and $I_{b}$ are both divergent when evaluated on solutions, as is the Hamiltonian, and other associated thermodynamic quantities [10, 11]. Rather than eliminating these divergences by incorporating a reference term in the spacetime [11, 23], a new term $I_{c t}$ is added to the action which is a functional only of boundary curvature invariants. Although there are a very large number of possible invariants one could add in a given dimension, only a finite number of them can eliminate the divergences at spatial infinity, which arise from $I_{v}$ and $I_{b}$. For an asymptotically AdS spacetime, these can be determined by an algorithmic procedure [18. Quantities such as energy, entropy, etc., can then be understood as intrinsically defined for a given spacetime, as opposed to being defined relative to some abstract (and non-unique) background, although this latter option is still available. In four dimensions the algorithm yields

$$
I_{c t(A d S)}=\frac{2}{l} \frac{1}{8 \pi} \int_{\partial \mathcal{M}_{\infty}} d^{3} x \sqrt{-\gamma}\left(1+\frac{l^{2}}{4} \mathcal{R}(\gamma)\right),
$$

where $l^{2}=3 /|\Lambda|$ and the coefficients have been chosen [5, 8, 18] so that the total action $I=$ $I_{v}+I_{b}+I_{c t(A d s)}$ is finite. We shall study the implications of including the terms in (3) for Kerr-AdS black holes in spatially finite regions. Although other counterterms (of higher mass dimension) may be added to $I_{c t}$, they will make no contribution to the evaluation of the action or Hamiltonian due to the rate at which they decrease toward spatial infinity, and we shall not consider them in our analysis here.

A generalization of the prescription (3) to spacetimes that are not asymptotically AdS is [5, 8]

$$
I_{c t}=\frac{2}{l} \frac{1}{8 \pi} \int_{\partial \mathcal{M}_{\infty}} d^{3} x \sqrt{-\gamma} \sqrt{1+\frac{l^{2}}{2} \mathcal{R}(\gamma)},
$$

which is equivalent to Eq. (3) for small $l$ (as well as fixed $l$ and large mean boundary radius), and which (formally) has a well defined limit for vanishing cosmological constant $(l \rightarrow \infty)$. A similar formula, in which $\mathcal{R}(\gamma)$ is replaced with the Ricci scalar of a large nearly spherical 2-surface embedded in $\Sigma$ was proposed by Lau [26]. We shall consider the implications of using Eq. (4) for Kerr black holes in spatially finite regions in the $l \rightarrow \infty$ limit. As in the asymptotically AdS case, although there are terms of higher mass dimension that could be included, we shall not consider these, since Eq. (4) is sufficient to remove divergences at spatial infinity for this case. An algorithmic procedure, developed for asymptotically flat cases yields Eq. (四), although there are topological restrictions on its applicability [18.

A thorough discussion of the quasilocal formalism has been given elsewhere [10, 11, 23] and so we only briefly recapitulate it here. The action can be written as a linear combination of a 
volume term Eq. (1), a boundary term Eq. (2) and a counterterm which we shall take to be Eq. (41) in either the small- $l$ or $l \rightarrow \infty$ limits:

$$
I=-\frac{1}{16 \pi}\left\{\int_{\mathcal{M}} d^{4} x \sqrt{-\gamma} \mathcal{R}+2 \int_{\partial \mathcal{M}} d^{3} x \sqrt{-\gamma} \Theta-\int_{\partial \mathcal{M}} d^{3} x \mathcal{L}_{\mathrm{ct}}\right\}
$$

Under the variation of the metric $\gamma$, one obtains:

$\left.\delta I=[\text { terms that vanish when the equations of motion hold }]^{\mu \nu} \delta g_{\mu \nu}+\int_{\partial \mathcal{M}} d^{3} x\left(P^{i j}+Q^{i j}\right)\right] \delta \gamma_{i j}$

where

$$
\begin{aligned}
P^{i j} & =\frac{\sqrt{-\gamma}}{16 \pi}\left(\Theta \gamma^{i j}-\Theta^{i j}\right), \\
Q^{i j} & =\frac{\sqrt{-\gamma}}{16 \pi} \frac{\partial \mathcal{L}_{\mathrm{ct}}}{\partial \gamma_{i j}}
\end{aligned}
$$

and Latin letters are used as indices for tensors on hypersurfaces.

Decomposing the metric $\gamma_{i j}$ on the timelike boundary $\partial \Sigma_{\tau} \times \Upsilon=\mathcal{B} \times \Upsilon=\mathcal{T}$ which connects the initial and final hypersurfaces

$$
\gamma_{i j} d x^{i} d x^{j}=-N^{2} d t^{2}+\sigma_{a b}\left(d x^{a}+V^{a}\right)\left(d x^{b}+V^{b}\right)
$$

yields after some manipulation

$$
\left.\delta I\right|_{\mathcal{T}}=\int_{\mathcal{T}} d^{3} x \sqrt{\sigma}\left(-\varepsilon \delta N+j_{a} \delta V^{a}+\frac{1}{2} N s^{a b} \delta \sigma_{a b}\right)
$$

where the coefficients of the varied fields are

$$
\begin{aligned}
\varepsilon & =\frac{2}{N \sqrt{\sigma}}\left(P^{i j}+Q^{i j}\right) u_{i} u_{j}, \\
j_{a} & =-\frac{2}{N \sqrt{\sigma}} \sigma_{a i}\left(P^{i j}+Q^{i j}\right) u_{j}, \\
s^{a b} & =\frac{2}{N \sqrt{\sigma}} \sigma_{i}^{a} \sigma_{j}^{b}\left(P^{i j}+Q^{i j}\right),
\end{aligned}
$$

and we see that the counterterm variation $Q^{i j}$ supplants terms which come from a reference action in the original formulation of the quasilocal technique. The standard assumption [10] for the reference action is that it is a linear functional of the lapse $N$ and shift $V^{a}$ so that its contributions to $\varepsilon$ and $j_{a}$ depend only on the induced two-metric $\sigma_{a b}$, whose determinant we denote by $\sigma$. This assumption does not hold for the counterterm prescription (4).

Since $-\sqrt{\sigma} \varepsilon$ is the time rate of change of the action, $\varepsilon$ is identified with the energy density on the surface $\mathcal{B}$, and the total quasilocal energy for the system is therefore

$$
E=\int_{\mathcal{B}} d^{2} x \sqrt{\sigma} \varepsilon
$$


and this quantity can be meaningfully associated with the thermodynamic energy of the system [24]. Using similar reasoning, the quantities $j_{a}$ and $s^{a b}$ are respectively referred to as the momentum surface density and the spatial stress.

When there is a Killing vector field $\xi$ on the boundary $\mathcal{T}$, an associated conserved charge is defined by

$$
\mathcal{Q}(\xi)=\int_{\mathcal{B}} d^{2} x \sqrt{\sigma}\left(\varepsilon u^{i}+j^{i}\right) \xi_{i}
$$

provided there is no matter stress energy in the neighborhood of $\mathcal{T}$ (this assumption can be dropped, allowing one to compute the time rate of change of $\mathcal{Q}$, if desired [25]). When this holds, the value of $\mathcal{Q}$ is independent of the particular hypersurface $\mathcal{B}$, a property not shared by the energy $E$. For boundaries with timelike $(\xi=\partial / \partial t)$ and rotational $(\varsigma=\partial / \partial \phi)$ Killing vector fields (which encompass all the metrics we consider in this paper) we obtain

$$
\begin{aligned}
M & =\int_{\mathcal{B}} d^{2} x \sqrt{\sigma}\left(\varepsilon u^{i}+j^{i}\right) \xi_{i} \\
J & =\int_{\mathcal{B}} d^{2} x \sqrt{\sigma} j^{i} \varsigma_{i}
\end{aligned}
$$

provided the surface $\mathcal{B}$ contains the orbits of $\varsigma$. These quantities are respectively the conserved mass and angular momentum of the system enclosed by the boundary. Note that they will both be dependent upon the location of the boundary $\mathcal{B}$ in the spacetime, although each is independent of the particular choice of foliation $\mathcal{B}$ within the surface $\mathcal{T}$.

In the context of the AdS/CFT correspondence the limit in which the boundary $\mathcal{B}$ becomes infinite is taken, and the counterterm prescription [14, 15, 16, 18] ensures that the conserved charges (15) are finite. No embedding of the surface $\mathcal{T}$ into a reference spacetime is required. This is of particular advantage for the class of Kerr spacetimes, in which it is not possible to embed an arbitrary two-dimensional boundary surface into a flat (or constant-curvature) spacetime 13 . The counterterm (4) includes the minimal number of boundary curvature invariants for which this holds in both the large- $l$ and $\mathcal{O}(l)$ limits. We shall consider the extension of Eq. (田) away from the $\mathcal{B} \rightarrow \infty$ limit in what follows.

The class of metrics we shall henceforth consider are the Kerr-AdS family of solutions, whose general form is

$$
\begin{aligned}
d s^{2}= & -\frac{\Delta_{r}}{\rho^{2}}\left(d t-\frac{a}{\Xi} \sin ^{2} \theta d \phi\right)^{2}+\frac{\rho^{2}}{\Delta_{r}} d r^{2}+\frac{\rho^{2}}{\Delta_{\theta}} d \theta^{2} \\
& +\frac{\Delta_{\theta} \sin ^{2} \theta}{\rho^{2}}\left[a d t-\frac{\left(r^{2}+a^{2}\right)}{\Xi} d \phi\right]^{2}
\end{aligned}
$$

in $(3+1)$ dimensions, where

$$
\begin{aligned}
\Delta_{r} & =\left(r^{2}+a^{2}\right)\left(1+r^{2} / l^{2}\right)-2 m r \\
\Delta_{\theta} & =1-a^{2} \cos ^{2} \theta / l^{2} \\
\Xi & =1-a^{2} / l^{2} \\
\rho^{2} & =r^{2}+a^{2} \cos ^{2} \theta
\end{aligned}
$$


and where $\Lambda=-3 / l^{2}$. The metric has two horizons located at $r_{ \pm}$, provided the parameter $m$ is sufficiently large relative to the other parameters, specifically

$$
m \geq \frac{l}{3 \sqrt{6}}\left(\sqrt{1+14 \frac{a^{2}}{l^{2}}+\frac{a^{4}}{l^{4}}}+2\left(1+\frac{a^{2}}{l^{2}}\right)\right) \sqrt{\sqrt{1+14 \frac{a^{2}}{l^{2}}+\frac{a^{4}}{l^{4}}}-\left(1+\frac{a^{2}}{l^{2}}\right)} \equiv m_{\text {crit }}
$$

In the limit $l \rightarrow \infty, r_{ \pm}=m \pm \sqrt{m^{2}-a^{2}}$, and $m_{\text {crit }}=a$.

For $m=0$, the metric (18) is that of pure AdS spacetime (or flat spacetime if $l \rightarrow \infty$ ), and for $a=0$, the metric is that of Schwarzchild-AdS spacetime which has zero angular momentum. Hence we expect the parameters $a$ and $m$ to be associated with the mass and angular momentum of the spacetime, respectively. This can be confirmed using conformal completion methods [27, for which we find that the total mass $M_{t}$ and angular momentum $J_{t}$ are given by 28]

$$
M_{t}=\frac{m}{\Xi} \quad, \quad J_{t}=\frac{a m}{\Xi^{2}},
$$

a result corroborated by counterterm methods [8].

We turn now to consider the extension of (4) away from the $\mathcal{B} \rightarrow \infty$ limit.

\section{Kerr Metric}

Here we take $l \rightarrow \infty$, and so the action can be written as

$$
I=-\frac{1}{16 \pi}\left\{\int_{\mathcal{M}} d^{4} x \sqrt{-\gamma} \mathcal{R}+2 \int_{\partial \mathcal{M}} d^{3} x \sqrt{-\gamma} \Theta-2 \sqrt{2} \int_{\partial \mathcal{M}} d^{3} x \sqrt{-\gamma} \sqrt{\mathcal{R}(\gamma)}\right\}
$$

We write, for convenience, $Q^{i j}=Q_{2}^{i j}+Q_{3}^{i j}$ where

$$
\begin{aligned}
Q_{2}^{i j} & =\frac{\sqrt{-\gamma}}{16 \pi} \sqrt{\frac{2}{\mathcal{R}}}\left(R^{i j}-\mathcal{R} \gamma^{i j}\right) \\
Q_{3}^{i j} & =\frac{\sqrt{-\gamma}}{16 \pi} \frac{1}{\sqrt{2}}\left(\nabla_{a}\left(\nabla^{a} \mathcal{R}^{-1 / 2}\right) \gamma^{i j}-\frac{1}{2} \nabla^{(i}\left(\nabla^{j)} \mathcal{R}^{-1 / 2}\right)\right)
\end{aligned}
$$

and the energy of the system can be written as :

$$
E=E_{1}+E_{2}+E_{3} ; \quad E_{i}=\int_{\mathcal{B}} d^{2} x \sqrt{\sigma} \varepsilon_{i},
$$

where $\mathcal{B}$ is a 2 -dimensional surface defined by setting the radial coordinate to a constant value $r=R$, and $\varepsilon_{1}, \varepsilon_{2}, \varepsilon_{3}$ are given by

$$
\begin{aligned}
\varepsilon_{1} & =\frac{2}{N \sqrt{\sigma}} P^{i j} u_{i} u_{j} \\
& =\frac{1}{4} R \frac{\sqrt{1+A^{2}-2 M}\left[(M-1) A^{2} z^{2}-2-(1+M) A^{2}\right]}{\left\{\left(1+A^{2} z^{2}\right)\left[\left(1+A^{2}-2 M\right) A^{2} z^{2}+1+A^{2}+2 M A^{2}\right]\right\}^{1 / 2}}
\end{aligned}
$$




$$
\begin{aligned}
\varepsilon_{2}= & \frac{2}{N \sqrt{\sigma}} Q_{2}^{i j} u_{i} u_{j} \\
= & \frac{1}{2} R\left\{\left(A^{4}-2 M\right) A^{4} z^{4}+\left(2 A^{2}-7 M A^{2}+2-4 M A^{4}-7 M\right) A^{2} z^{2}\right. \\
& \left.+1+A^{2}+3 M A^{2}+A^{4} M\right\} \\
& \times\left\{\left(1-2 M A^{2} z^{2}+A^{2} z^{2}\right)\left[\left(1+A^{2}-2 M\right) A^{2} z^{2}+1+2 M A^{2}+A^{2}\right]\left(1+A^{2} z^{2}\right)^{3}\right\}^{-1 / 2} \\
\varepsilon_{3}= & \frac{2}{N \sqrt{\sigma}} Q_{3}^{i j} u_{i} u_{j} \\
= & \frac{1}{2} R\left\{\left(1-2 M^{2}\right)\left(1+A^{2}-2 M\right) A^{8} z^{10}\right. \\
& +(1-2 M)\left(12 M^{2}-4 A^{2} M^{2}-24 M-6 M A^{2}+9+9 A^{2}\right) A^{6} z^{8} \\
& +\left[(2 M-1)^{3} A^{4}-\left(36 M^{3}-42 M^{2}-7 M+14\right) A^{2}+(2 M-1)\left(2 M^{2}+15 M-15\right)\right] A^{4} z^{6} \\
& +\left[-3(2 M-1)(M-1) A^{4}-\left(8 M^{3}+36 M^{2}-25 M-8\right) A^{2}-8 M^{2}-12 M+11\right] A^{2} z^{4} \\
& +\left(3-8 A^{4} M^{3}-3 A^{4}+3 M+15 A^{2} M+4 A^{4} M^{2}+6 A^{2} M^{2}\right) z^{2} \\
& \left.-1-M-A^{2}-2 A^{2} M^{2}-3 A^{2} M\right\} \\
& \times\left\{\left(1-2 M A^{2} z^{2}+A^{2} z^{2}\right)^{5}\left[\left(1+A^{2}-2 M\right) A^{2} z^{2}+1+2 M A^{2}+A^{2}\right]\left(1+A^{2} z^{2}\right)^{3}\right\}^{-1 / 2}
\end{aligned}
$$

where $z=\cos \theta$ and $A=a / R$ and $M=m / R$.

For arbitrary values of $A$ and $M$, the term $E_{1}$ can be integrated analytically in terms of Elliptic functions. Although the terms $E_{2}$ and $E_{3}$ cannot be analytically integrated, a term-by-term expansion of $E_{3}$ in powers of $A$ indicates that it is zero, a fact that can also be verified numerically.

For small angular momentum $A, E$ can be integrated easily:

$$
\left.E=R\left\{1-\sqrt{1+A^{2}-2 M}+\frac{A^{2}}{6}\left[2(1+M)+(1+2 M) \sqrt{1+A^{2}-2 M}\right]+O\left(A^{4}\right)\right]\right\} .
$$

In the asymptotic limit $R \rightarrow \infty$,the energy approaches the Arnowitt-Deser-Misner (ADM) energy $M$, and for the case of $A=0$, one gets the thermodynamic energy of a Schwarzschild black hole, which is equivalent to its mass. The expression (29) was obtained by Martinez [13] using the quasilocal method with a flat reference spacetime in which the constant- $R$ boundary was embedded in the small- $A$ limit. However even in this limit the embedding was technically quite complicated. We find it interesting that the counterterm prescription (22), extended to finite values of fixed $R$, gives exactly the same answer to this order in $A$.

We next consider the total energy $E$ in (25) for $R=r_{+}$where $r_{+}$is the radius of the outer horizon. In this case we can exactly compute $E$ in terms of elliptic functions:

$$
E\left(r_{+}\right)=-\frac{1+A_{+}^{2}}{2} r_{+}\left[\left(4+\frac{3}{A_{+}^{2}}\right) F\left(A_{+}^{2}, \frac{i}{A_{+}}\right)-5 E\left(A_{+}^{2}, \frac{i}{A_{+}}\right)-5 \sqrt{1-A_{+}^{2}}\right]
$$

where $i^{2}=-1, A_{+}=a / r_{+}$, and

$$
E(z, k)=\int_{0}^{z} d t \frac{\sqrt{1-k^{2} t^{2}}}{\sqrt{1-t^{2}}} \quad F(z, k)=\int_{0}^{z} d t \frac{1}{\sqrt{1-t^{2}} \sqrt{1-k^{2} t^{2}}}
$$




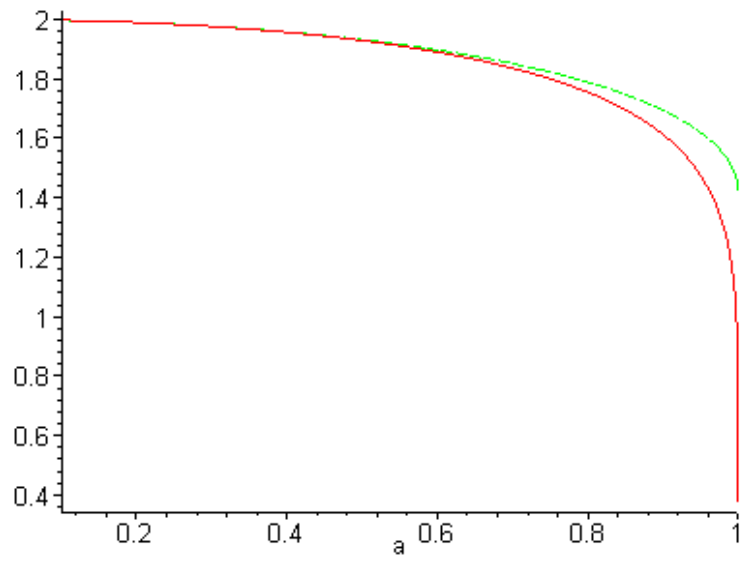

Figure 1: $E\left(r_{+}\right) / m$ (solid), and $2 M_{i} / m$ (dashed) versus a plotted in units of $m$.

are the definitions of the elliptic functions $E(z, k)$ and $F(z, k)$.

In Fig.. 1 we plot the $A_{+}$-dependence of the energy at the horizon. In the slow-rotation approximation it has been shown that

$$
E\left(r_{+}\right)=2 M_{i}\left[1+\mathcal{O}\left(\frac{a^{4}}{M_{i}^{4}}\right)\right],
$$

where

$$
M_{i}=\frac{1}{2} \sqrt{r_{+}^{2}+a^{2}}
$$

is the irreducible mass of the Kerr black hole [29], the maximal amount of energy which can be extracted from a black hole via the Penrose process. It was conjectured in Ref. [13 that the relation $E\left(r_{+}\right)=2 M_{i}$ is an exact relation, valid for any value of $A$. We see using the counterterm prescription that this conjecture is not correct and that (31) is only an approximation which breaks down for $\frac{a}{R}>0.5$, as shown in Figs. 1 and 2 . 


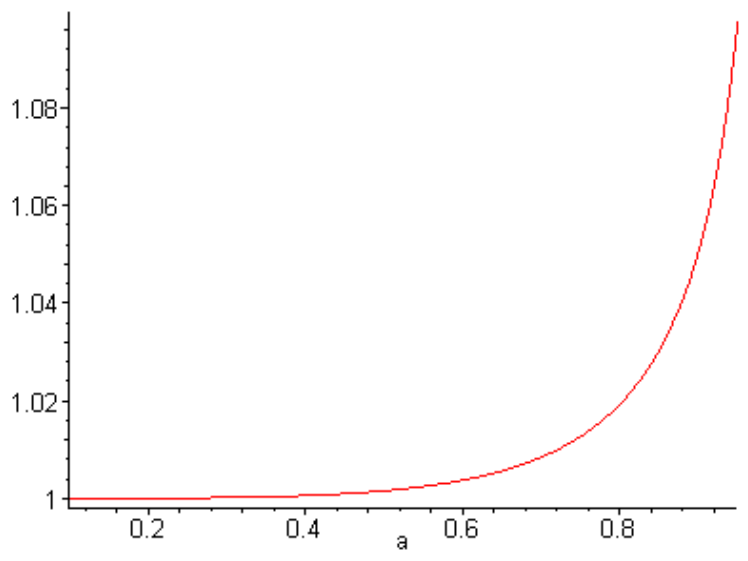

Figure 2: $M_{i} / E\left(r_{+}\right)$versus a plotted in units of $m$. 


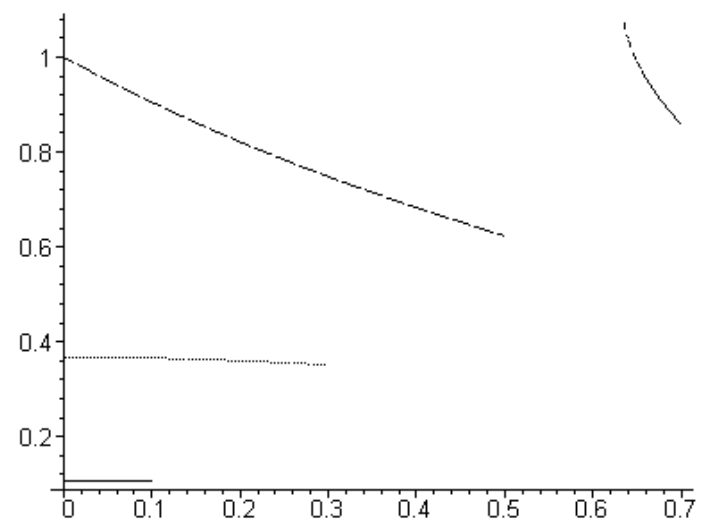

Figure 3: $E / R$ versus $A$ for $M=0.1$ (solid), 0.3 (dotted), 0.5 (dashed), 0.7 (dot-dashed).

We next consider evaluation of the energy for fixed arbitrary values of $R$. The calculation of $E_{2}$ must be carried out numerically, and in a manner which yields a well defined integral. This means that the parameters of the integral must be such that the location of the quasilocal surface must be outside of the event horizon and the induced Ricci scalar $\mathcal{R}(\gamma)$ must be positive. These criteria are satisfied provided that $A$ and $M$ are such that :
a) For $M \leq 0.5$ then $0 \leq A \leq M$,
b) For $0.5 \leq M \leq 1$ then $\sqrt{2 M-1} \leq A \leq M$.
c) $A \leq 1, M \leq 1$

We plot the dependence of $E(M, A)$ in Figs. 3 and 1 below, consistent with these constraints. As one can see from Fig. 3, for small values of $M(M<0.4)$ the energy is a weakly varying function of $A$ but as $M$ increases the $A$-dependence of energy becomes more relevant. For example the average value of $|\partial E / \partial A|$ is approximately $10^{-2}$ and 10 for $M=0.1$ and $M=0.8$ respectively. Of course these values of $M=m / R$ depend on the value of the radius $R$ which is chosen for the quasilocal surface. 


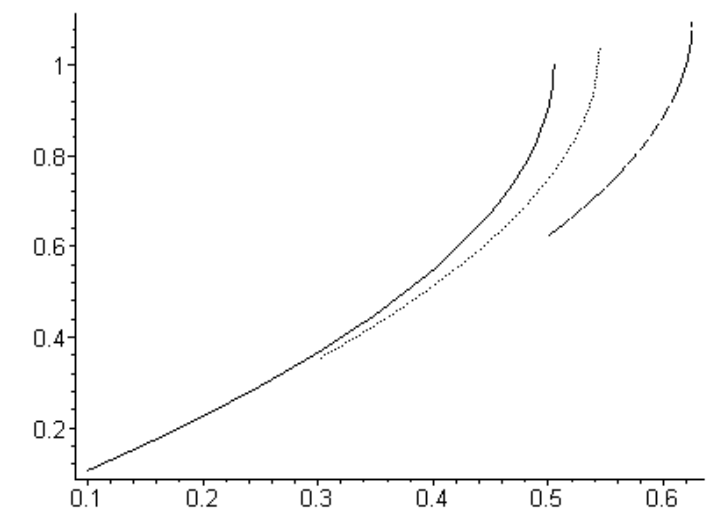

Figure 4: $E / R$ versus $M$ for $A=0.1$ (solid), 0.3 (dotted), 0.5 (dashed). 
Now we consider the quasilocal conserved charges of the Kerr metric. The Kerr metric has two Killing vectors and therefore there exist two conserved quantities. The first one, which is due to the timelike Killing vector $\xi^{a}=(\partial / \partial t)^{a}$, is associated with the conserved mass, $\mathcal{M}$, of the system expressed as:

$$
\mathcal{M}=-\int_{B} d^{2} x \sqrt{\sigma} \frac{2}{N}\left(P^{i j}+Q_{2}^{i j}+Q_{3}^{i j}\right) u_{i} \xi_{j}
$$

where $N$ is the lapse function. For the case of $R=r_{+}$, we can explicitly compute the conserved mass:

$$
\mathcal{M}\left(r_{+}\right)=\frac{1}{2} r_{+} A^{2}=\frac{a^{2}}{2 r_{+}}=m-\frac{1}{2} r_{+}=\frac{1}{2} r_{-}
$$

which should be compared with the expression for the quasilocal energy. In the limit of slow rotation it vanishes, a strikingly different feature from that of the energy as given in eq. (31), which in this limit equals $2 m$. As $R \rightarrow \infty$ we find that $M \rightarrow m$ as expected.

It is clear from Figs. 3 and 4 that the energy for a fixed value of $A$ is an increasing function of $M$. This is not the case for the conserved mass $\mathcal{M}(M, A)$ in (34), which for fixed $A<0.5$ has a maximum value for $M \geq \frac{1}{3}$, with the bound being saturated for the Schwarzchild solution when $A=0$. We plot in Figs. 5 and 6 the behavior of the conserved charge $\mathcal{M}(M, A)$ for fixed $A$ and $M$ respectively. We find that the value of $\mathcal{M}$ due to the $Q_{3}^{i j}$ third term in eq. (34) is very small compared to that of the first two terms.

The second non-trivial conserved charge is the $\varphi$ component of the angular momentum, $J_{\varphi}$, which can be expressed as :

$$
J_{\varphi}=-\int d^{2} x \sqrt{\sigma} \frac{2}{N}\left(P^{i j}+Q_{2}^{i j}+Q_{3}^{i j}\right) u_{i} \sigma_{j a} \zeta^{a}
$$

where $\zeta^{a}$ is Killing vector $\left(\frac{\partial}{\partial \varphi}\right)$. Remarkably we find that the counter terms $Q_{2}^{i j}+Q_{3}^{i j}$, while nonzero, give a vanishing contribution upon integration in (36). Hence the total angular momentum is due entirely to the first term, which is:

$$
J_{\varphi}=r^{2} M A=m a
$$

valid for any quasilocal surface of fixed $r=R$.

We turn next to thermodynamic considerations of the Kerr black hole. The interior of the quasilocal surface can be regarded as a thermodynamic system which is co-rotating with an exterior heat bath whose angular velocity is $\Omega_{H}$ [30], where $\Omega_{H}=\frac{a}{r_{+}^{2}+a^{2}}$ is the angular velocity of the event horizon. Zero angular momentum observors (ZAMO's) at a given position outside of the black hole measure a proper inverse temperture $\beta_{\mathrm{ZAMO}}=N \beta$ and a proper angular velocity $\Omega_{\mathrm{ZAMO}}(r, \theta)=N^{-1}\left(\Omega_{H}-\frac{d \phi}{d t}\right)$. However quasilocal thermodynamic quantities are generally given by surface integrals over the boundary data rather than by products of constant surface data. In particular, it is not possible to choose the quasilocal surface to simultaneously be both isothermal and a surface of constant $\Omega$ ZAMO and neither is true for the quasilocal surface we have chosen (one at fixed $r=R$ ). Furthermore it is by now well recognized that the entropy of 


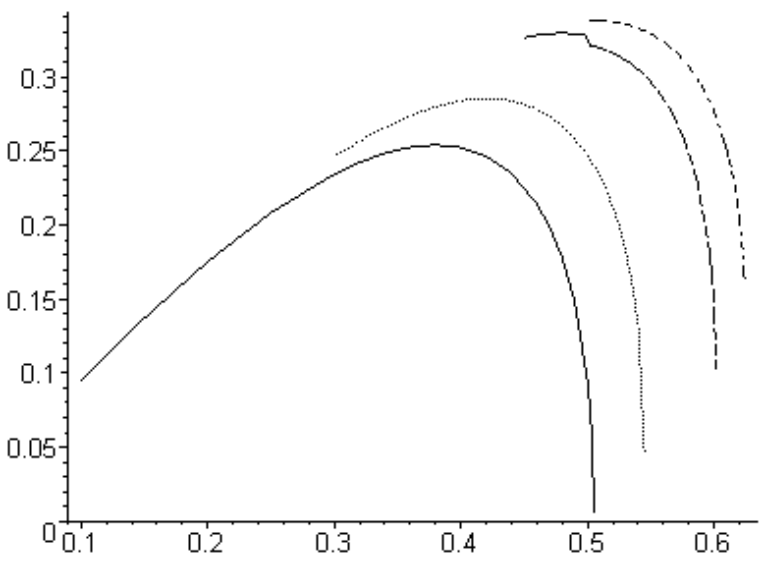

Figure 5: $\mathcal{M} / R$ versus $M$ for $A=.1$ (solid), .3 (dotted), 0.45 (dashed), 0.5 (dot-dashed). 


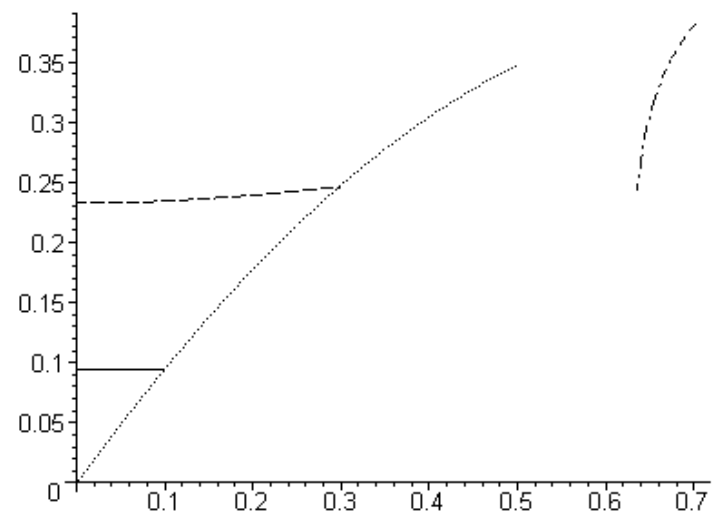

Figure 6: $\frac{\mathcal{M}}{R}$ versus $A$ for $M=.1$ (solid), 0.3 (dotted), 0.5 (dashed), 0.7 (dot-dashed). 
a black hole depends only on the geometry of its horizon in the classical approximation, and is conversely independent of the asymptotic behavior of the gravitational field or of the presence of external matter fields. For stationary black holes, the entropy is one-quarter of the horizon area, yielding $S=\pi\left(r_{+}^{2}+a^{2}\right)=2 \pi R^{2} M\left(M+\sqrt{M^{2}-A^{2}}\right)$ in this case. We therefore shall regard the energy $E$ in Eq. (25) as the thermodynamic internal energy for the Kerr black hole within the boundary $r=R$, view it as a function of $R$, the entropy $S$, and the angular momentum $J$, and define the temperature and the angular velocity from their ensemble derivatives, integrated over the quasilocal boundary data.

For the temperature we obtain

$$
T=\left(\frac{\partial E}{\partial S}\right)_{R, J}=\frac{1}{4 \pi R} \frac{\sqrt{M^{2}-A^{2}}}{M\left(M+\sqrt{M^{2}-A^{2}}\right)}\left(\frac{\partial E}{\partial M}\right)_{R, J}=\frac{\kappa_{+}}{2 \pi R}\left(\frac{\partial E}{\partial M}\right)_{R, J}
$$

where $\kappa_{+}$is the surface gravity at the outer horizon. The derivative $\left(\frac{\partial E}{\partial M}\right)_{R, J}$ is obtained by taking the derivatives of the terms in Eqs. (26-28) above and then performing the integration with respect to $z=\cos \theta$. It is straightforward to show that $(\partial E / \partial M)_{R, J} \rightarrow 1$ as $R \rightarrow \infty$, yielding the usual relationship between temperature and surface gravity. However unlike the non-rotating case, it does not give the Tolman redshift factor because a surface of fixed $r=R$ is not a surface of constant redshift (it is not an isotherm).

Now we investigate the $A$ and $M$ dependence of the temperature of a Kerr black hole. The $A$ dependence of the temperature is shown in Fig. 7. For $M<0.4$ the temperature does not depend on $A$ very much (except for $(M-A) / M \ll 1$, where $T \longrightarrow 0$ as $A \rightarrow M$ ), but as $M$ increases ( $M \geq 0.5)$ the $A$-dependence of $T$ becomes more relevant. For the case of $M>0.5$ as $r \rightarrow r_{+}$then $T$ goes to infinity and it goes to zero as $A$ goes to $M$. The $M$-dependence of $T$ is shown in fig. 8 . The $A$-dependence of $\left(\frac{\partial T}{\partial M}\right)_{J, R}$ can be seen from fig. 9.

The temperature for a fixed value of $J$ does not increase monotically. For example from fig. 9 for $A=0.1$, it can be seen that $(\partial T / \partial M)_{J, R}$ is zero at $M \simeq 0.16$, and $M \simeq 0.32$. These two points correspond to the maximum and minimum of $T(M, J, R)$ respectively. $T(M, J, R)$ for the Schwarzschild case with $A=0$, has its minimum at $M=1 / 3$. For small values of $A$, the situation is similar; at a fixed value of the temperature $T$ below $T_{\text {min }}$ there will be only one black hole solution of small $M$, in equilibrium with thermal radiation inside the box of characteristic radius $r=R$, which will not be far from the extremal solution. At $T$ increases above $T_{\text {min }}$ two more possible solutions appear, one of slightly larger $M$ and one of much larger $M$. As $T$ increases above $T_{\max }$ the two smaller $M$ coalesce, leaving only the largest $M$ solution. As $A$ increases the region for the three distinct black hole solutions becomes vanishingly small, and for sufficiently large $A$, there will be only one black hole solution of large $M$.

The heat capacity at constant surface area, $4 \pi R$, is defined by:

$$
C_{R}=\left(\frac{\partial E}{\partial T}\right)_{R, J}
$$




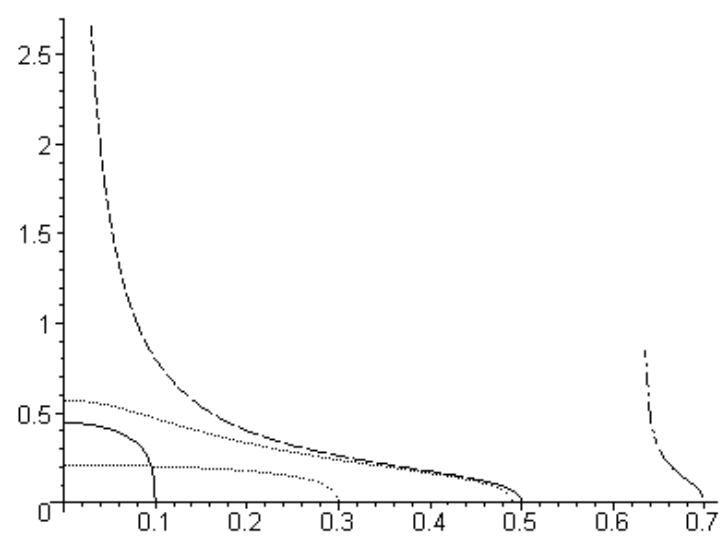

Figure 7: $R T$ versus $A$ for $M=0.1$ (solid), 0.3 (dotted), 0.49 (light dotted) 0.5 (dashed), .7 (dot-dashed). 


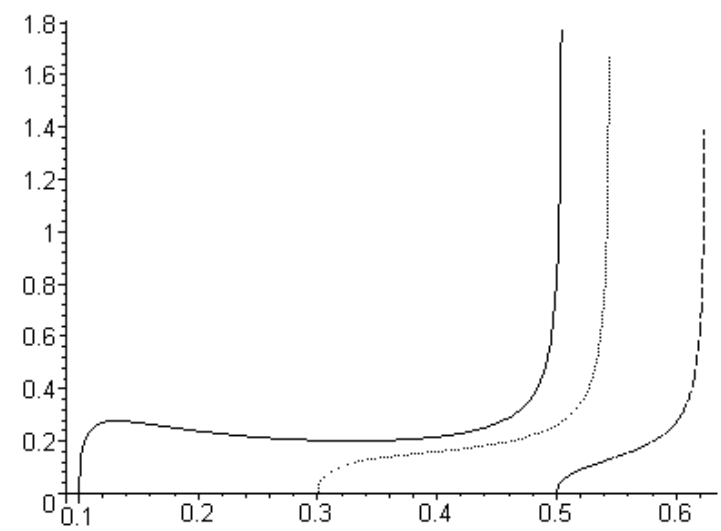

Figure 8: $R T$ versus $M$ for $A=0.1$ (solid), 0.3 (dotted), 0.5 (dashed). 


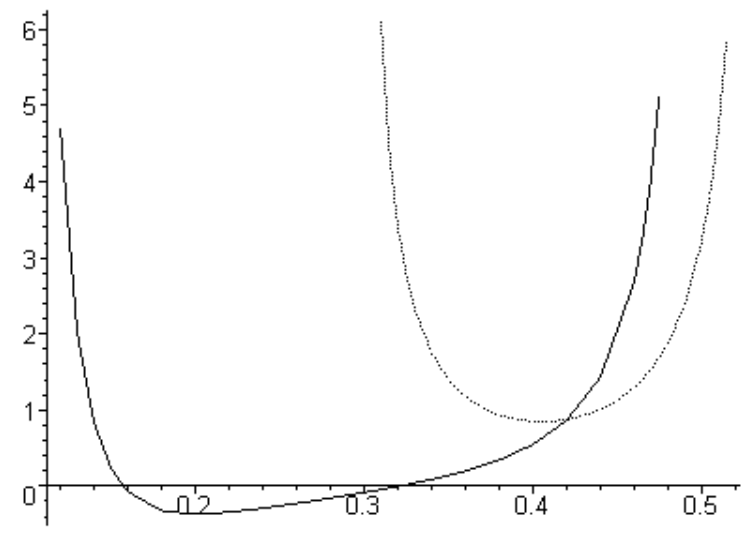

Figure 9: $R(\partial T / \partial M)$ versus $M$ for $A=0.1$ (solid), 0.3 (dotted). 
where the energy is expressed as a function of $T$ and $R$. Expressing the energy and temperature as functions of $M, J$ and $R$, then the heat capacity can be written as

$$
C_{R}=\left(\frac{\partial E}{\partial M}\right)_{R, J}\left(\frac{\partial T}{\partial M}\right)_{R, J}^{-1}
$$

For small values of $M(M<1 / 3)$, the heat capacity is not positive for all the allowed values of $A$. For example as one can see from fig. 10 for $M=0.1$, the heat capacity is positive only for $A>0.055$. Indeed, for each value of $M<1 / 3$ there exists an $A$ for which the heat capacity becomes infinite. For $M \approx 1 / 3$ the heat capacity is negative for a Schwarzschild black hole. However for a Kerr black hole we see from fig. ?? there are some values of $A$ for which the heat capacity is positive. There is a phase of stable small black holes and a phase of stable large black holes, separated by a regime of intermediate unstable black holes, as illustrated in fig. 9.

This fact was noted long ago by Davies, who gave the relation

$$
a^{4}+6 a^{2} m^{2}-3 m^{4}=0
$$

for the values of $a / m$ at which the heat capacity becomes infinite 21] in the limit $R \rightarrow \infty$. Here since we use the quasilocal energy at $r=R$ instead of the ADM mass parameters the relationship is considerably more complicated, and can only be obtained numerically. However the qualitative features remain the same. The $A$-dependence of the heat capacity for larger values of $M$ can be seen from Figs. 10 and 11. For sufficiently large $M$, the heat capacity is positive for all allowed values of $A$, and there is a single phase consisting of a large black hole.

Expressing the energy as a function of $R, S$ and $J$, the angular velocity can be written as

$$
\Omega=\left(\frac{\partial E}{\partial J}\right)_{S, R}=\left(\frac{\partial E}{\partial M}\right)_{S, R}\left(\frac{\partial M}{\partial J}\right)_{S, R}=\Omega_{H}\left(\frac{\partial E}{\partial M}\right)_{R, S}
$$

where $\Omega_{H}$ is the angular velocity at the event horizon. As $R \rightarrow \infty,(\partial E / \partial M)_{R, S} \rightarrow 1$ and thermodynamic chemical potential conjugate to the angular momentum approaches $\Omega_{H}$. The $A$ dependence of $\Omega$ is illustrated in Fig. 13 .

As expected, $\Omega$ decreases with decreasing $A$. However the derivative $(\partial E / \partial M)_{R, S}$ is not positive for all values of $A$ once $M$ becomes sufficiently large, or (alternatively) $R$ becomes sufficiently small. This suggests that some kind of superradiance will take place for sufficiently small quasilocal boundaries. The numerical computation required to extrapolate the curve for $M=0.5$ is more intense than the computational power we have available; however we have explicitly checked that $\Omega$ vanishes for small $A$ in this case. We shall not pursue this issue further. 


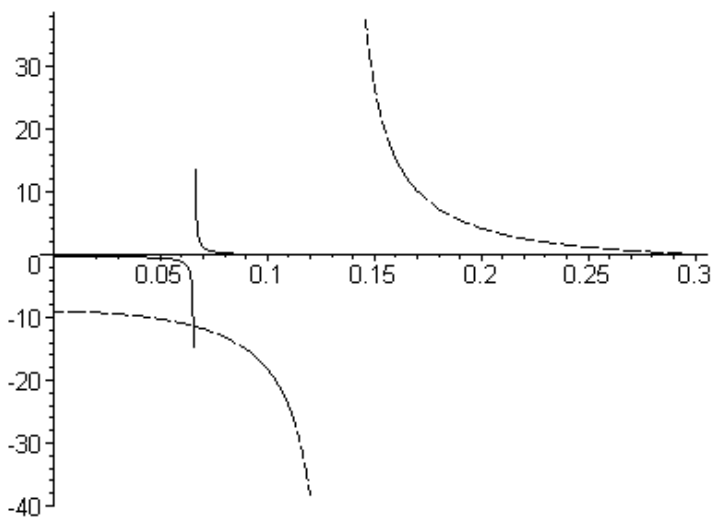

Figure 10: $C_{R} / R^{2}$ versus $A$ for $M=0.1$ (solid), 0.3 (dashed). 


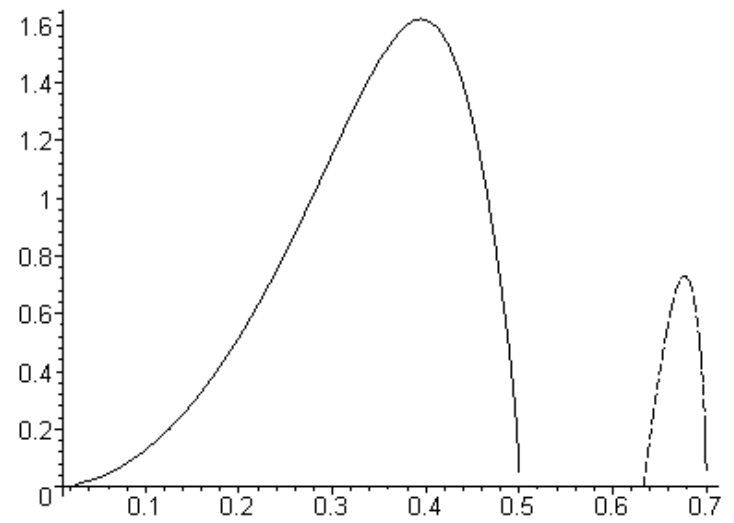

Figure 11: $C_{R} / R^{2}$ versus $A$ for $M=0.5$ (solid), 0.7 (dashed). 


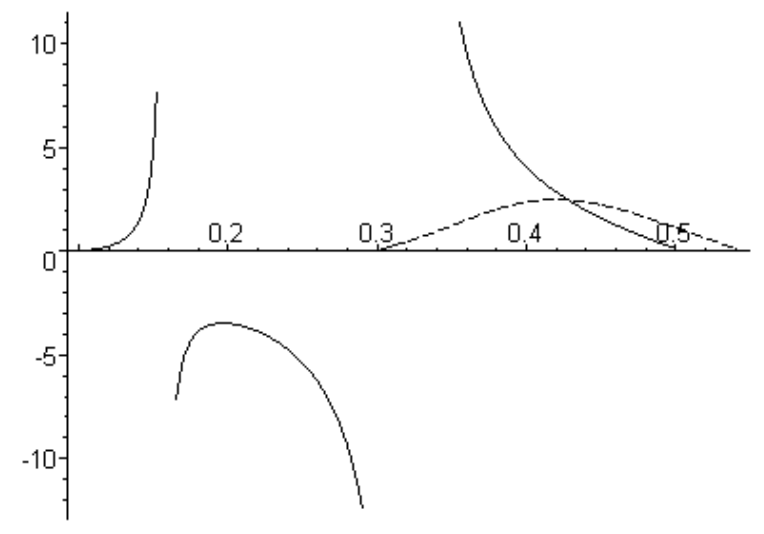

Figure 12: $C_{R} / R^{2}$ versus $M$ for $A=0.1$ (solid), 0.3 (dashed). 


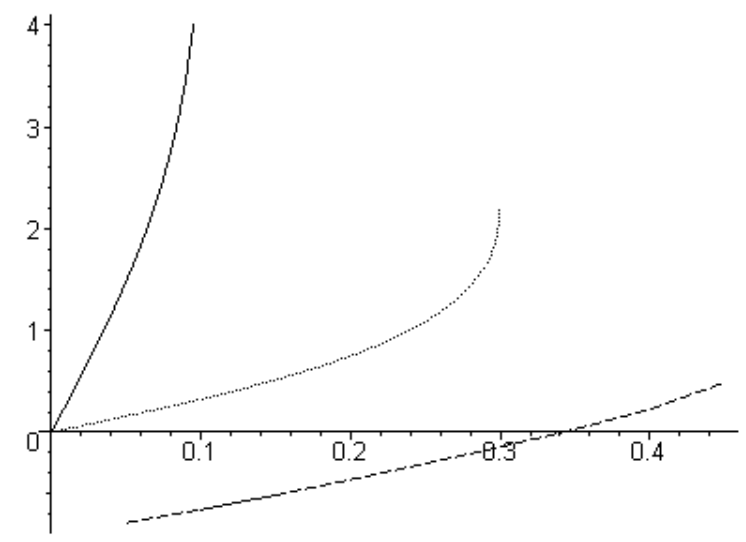

Figure 13: $R \Omega$ versus $A$ for $M=0.1$ (solid), 0.3 (dotted), 0.5 (dashed). 


\section{Kerr-AdS Metric}

For the Kerr-AdS metric the action can be written as a linear combination of a volume term, a boundary term and the counterterm (14) in the small- $l$ limit:

$$
I=-\frac{1}{16 \pi}\left\{\int_{\mathcal{M}} d^{4} x \sqrt{-\gamma}(\mathcal{R}-2 \Lambda)+2 \int_{\partial \mathcal{M}} d^{3} x \sqrt{-\gamma} \Theta-\frac{4}{l} \int_{\partial \mathcal{M}} d^{3} x \sqrt{-\gamma}\left(1+\frac{l^{2}}{4} \mathcal{R}(\gamma)\right)\right\}
$$

Under variation of the metric, $\gamma$, one obtains:

$\delta I=\frac{1}{16 \pi}\left\{[\right.$ terms that vanish when the equations of motion hold $\left.] \delta g_{i j}+\left[\int_{\partial \mathcal{M}} \sqrt{-\gamma}\left(P^{i j}+Q^{i j}\right)\right] \delta \gamma_{i j}\right\}$

where now

$$
Q^{i j}=\frac{1}{16 \pi}\left(l G^{i j}-\frac{2}{l} \gamma^{i j}\right)
$$

Therefore the energy of the system can be written as:

$$
E=E_{1}+E_{2} ; \quad E_{i}=\int_{B} d^{2} x \sqrt{\sigma} \varepsilon_{i},
$$

where $\varepsilon_{1}$ and $\varepsilon_{2}$ are given by

$$
\begin{aligned}
& \sqrt{\sigma} \varepsilon_{1}=\frac{2}{N} P^{i j} u_{i} u_{j} \\
& =\frac{L r \sqrt{1+L^{2}+A^{2} L^{2}+A^{2}-2 M L^{2}}}{4\left(L^{2}-A^{2}\right)\left(L^{2}-A^{2} z^{2}\right)\left(1+A^{2} z^{2}\right)} \\
& \times \frac{\left[\left(L^{2}-A^{2}-M L^{2}\right) A^{2} z^{2}-2 A^{2}+A^{2} L^{2}+A^{2} M L^{2}-A^{4}+2 L^{2}\right]}{\left\{\left(L^{2}+A^{2} L^{2}-A^{2}-2 M L^{2}-A^{4}\right) A^{2} z^{2}+A^{2} L^{2}-A^{2}-A^{4}+2 A^{2} M L^{2}+L^{2}\right\}^{1 / 2}} \\
& \sqrt{\sigma} \varepsilon_{2}=\frac{2}{N} Q^{i j} u_{i} u_{j} \\
& =\frac{L r}{4\left(L^{2}-A^{2}\right)\left(1+A^{2} z^{2}\right)^{3}}\left\{\left(L^{2}+A^{2} L^{2}-A^{4}-A^{2}-2 M L^{2}\right) A^{8} z^{8}\right. \\
& +\left(4 L^{2}-6 M L^{2}+2 M L^{2} A^{2}+4 L^{2} A^{2}-4 A^{2}-4 A^{4}\right) A^{6} z^{6} \\
& +\left(L^{4}+6 M L^{2} A^{4}+A^{2} L^{4}+6 A^{2} L^{2}-7 A^{2}-A^{4} L^{2}+22 M L^{2} A^{2}-4 M^{2} L^{4}\right. \\
& \left.-7 A^{4}+2 A^{2} L^{4} M+7 L^{2}+2 M L^{2}\right) A^{4} z^{4} \\
& +\left(4 A^{2} L^{2}-6 A^{2}-6 A^{4}-4 M L^{2}+4 A^{2} M^{2} L^{4}-8 A^{4} L^{4} M\right. \\
& \left.+6 L^{2}-4 M L^{2} A^{4}+2 A^{2} L^{4}+4 M L^{2} A^{2}-10 M L^{4}+2 L^{4}-14 M A^{2} L^{4}-2 A^{4} L^{2}\right) A^{2} z^{2} \\
& \left.+2 M A^{4} L^{4}+2 L^{2}-2 A^{2}-A^{4} L^{2}+L^{4} A^{2}+4 M L^{2} A^{2}+A^{2} L^{2}+L^{4}-2 A^{4}+4 M A^{2} L^{4}\right\} \\
& \times\left\{\left(L^{2}-A^{2} z^{2}\right)\left[\left(L^{2}+A^{2} L^{2}-A^{2}-2 M L^{2}-A^{4}\right) A^{2} z^{2}+A^{2} L^{2}-A^{2}-A^{4}+2 A^{2} M L^{2}+L^{2}\right]\right\}^{1 / 2}
\end{aligned}
$$

with $A=a / R, M=m / R$ and $L=l / R$.

For $A=0$, we recover the quasilocal energy of a Schwarzchild-AdS black hole [11. Following our previous approach, we first consider special cases in which the integral in Eq. (46) can be 
evaluated. For $R=r_{+}$, where $r_{+}$is the radius of the horizon, we find that the total energy can be explicitly computed, yielding

$$
E\left(r_{+}\right)=\frac{l^{2}+3 a^{2}+4 r_{+}^{2}}{2\left(l^{2}-a^{2}\right)} l
$$

For small values of angular momentum, $E$ can be integrated easily. Then the total energy for small $A$ is:

$$
E=\frac{R}{L}\left\{\frac{2+L^{2}}{2}+\frac{2}{3} A^{2}\left(1+M+\frac{1}{L^{2}}\right)-\sqrt{1+A^{2}-2 M+\frac{1+A^{2}}{L^{2}}}\left[1+\frac{A^{2}}{6}\left(1+2 M-\frac{4}{L^{2}}\right)\right]+O\left(A^{4}\right)\right\}
$$

When $A=0$ the energy is given by

$$
E=R\left(\frac{1}{L}+\frac{L}{2}-\sqrt{1+\frac{1}{L^{2}}-2 M}\right)
$$

which differs from the expression obtained using background subtraction methods [11.

For general values of the parameters, however, we cannot analytically integrate (46) exactly, and so we resort to numerical integration. To do this entails several restrictions on the parameters. First, the Kerr-AdS solution is defined only for $L>A$. Furthermore, since the quasilocal surface must be outside of the horizon, $r_{+} / r$ must be real and less than unity. Note that there is no longer a restriction on the sign of the induced Ricci scalar. These criteria yield this fact that if one choose two of the three parameters $M, L$ and $A$, then the allowed values of the third parameter should obeys the following equations:

$$
\begin{aligned}
& \text { for given values of } A \text { and } L, M_{\mathrm{crit}} \leq M \leq \frac{\left(1+A^{2}\right)\left(1+L^{2}\right)}{2 L^{2}} \\
& \text { for given values of } A \text { and } M, \quad L_{\mathrm{crit}} \leq L \leq \sqrt{\frac{\left(1+A^{2}\right)}{2 M-\left(1+A^{2}\right)}} \text {, if } 2 M>\left(1+A^{2}\right), \\
& \text { for given values of } M \text { and } L, \sqrt{\frac{2 M L^{2}}{1+L^{2}}-1} \leq A \leq A_{\mathrm{crit}} \text { if } 2 M>\left(1+L^{2}\right),
\end{aligned}
$$

where $L_{\text {crit }}=\frac{{ }^{l} \text { crit }}{R}, A_{\text {crit }}=\frac{{ }^{a} \text { crit }}{R}$, and $M_{\text {crit }}=\frac{{ }^{m_{\text {crit }}}}{R}$ given by (20). For $M \neq 0.5$ and $2 M<$ $1+A^{2}$ there is no upper bound for $L$, and for $2 M<\left(1+L^{2}\right)$, the lower bound for $A$ is zero.

In the next four figures we plot the quasilocal energy as a function of $A$ and $M$. The $A$ dependence of the energy is given in Figs. 14 and 15. We see that for small values of $L(L \sim 1)$ the energy is a slowly increasing function of $A$. For larger values of $L$, as in the case of Kerr metric, for $M<0.5$ the energy decreases as $A$ increases. 


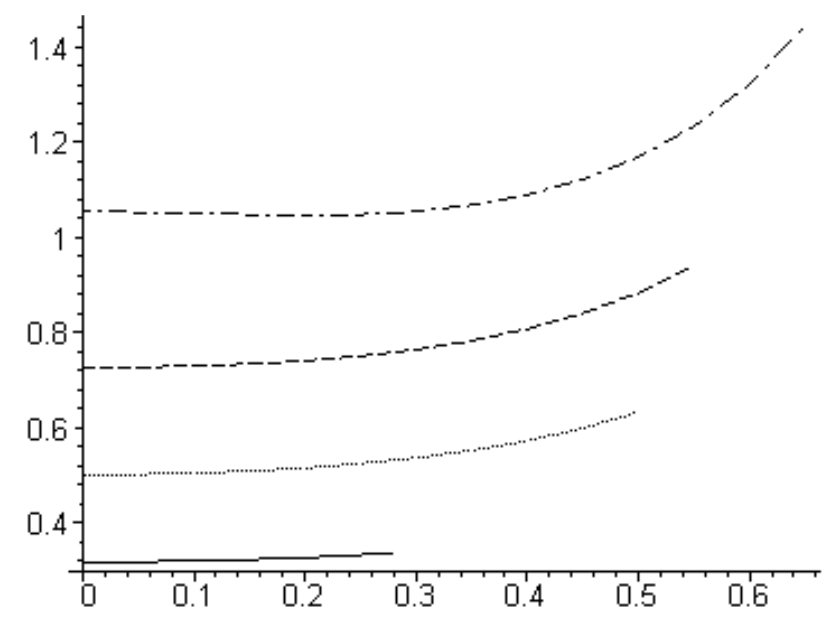

Figure 14: $E / R$ versus $A$ for $L=1, M=0.3$ (solid), 0.5 (dotted), 0.7 (dashed), 0.9 (dot-dashed). 


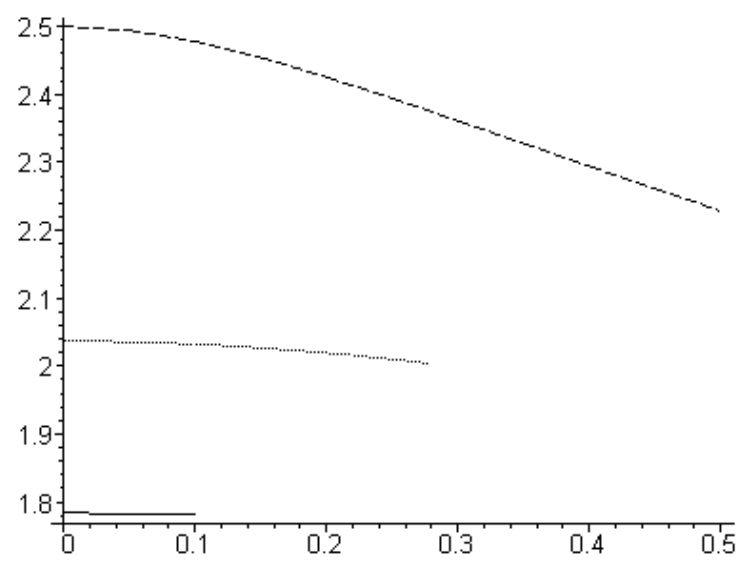

Figure 15: $E / R$ versus $A$ for $L=5, M=0.1$ (solid) 0.3 (dotted), 0.5 (dashed). 


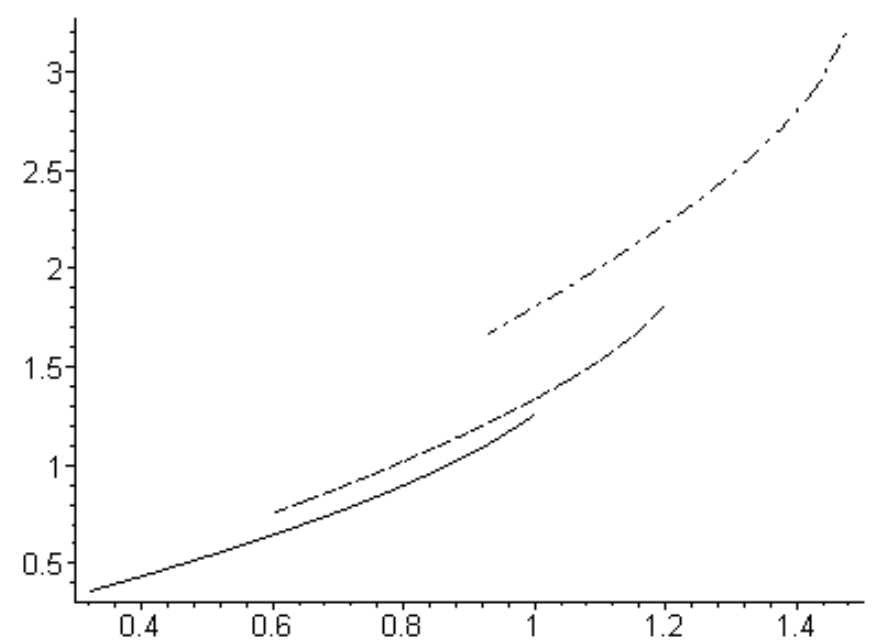

Figure 16: $E / R$ versus $M$ for $L=1, A=0.3$ (solid), 0.5 (dashed), 0.7 (dot-dashed). 


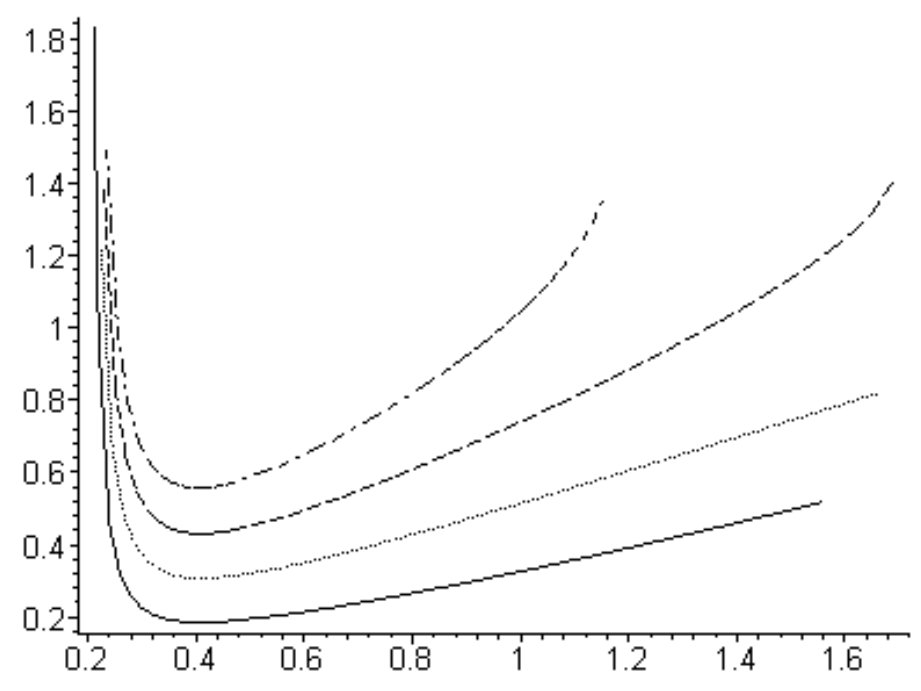

Figure 17: $E / R$ versus $L$ for $A=0.2, M=0.3$ (solid), 0.5 (dotted), .7 (dashed), 0.9 (dot-dashed). 
Figures 16 and 17 show the $M$-dependence and $L$-dependence of the energy. For large values of $L$ the energy is asymptotic to a linear function of $L$. This is due to the fact that the counterterm used for Kerr-AdS is proportional to $L$ for large values of $L$.

As in the case of Kerr metric we consider the quasilocal conserved charges of the Kerr-AdS metric which are the mass and $\varphi$ component of angular momentum of the system defined in eqns. (16,177). The $\varphi$ component of angular momentum due to the counterterm is zero and one can evaluate the total angular momentum as

$$
J_{\varphi}=\operatorname{am}\left(1-\frac{a^{2}}{l^{2}}\right)^{-2}
$$

which is therefore valid for any quasilocal surface of fixed $r=R$, and yields the angular momentum of the Kerr metric as $l$ goes to infinity.

The total mass of the system, $\mathcal{M}$, is somewhat more complicated to evaluate. For $R=r_{+}$it is

$$
\mathcal{M}\left(r_{+}\right)=\frac{a^{2}}{2 r_{+}}\left(1+\frac{r_{+}^{2}}{l^{2}}\right)\left(1-\frac{a^{2}}{l^{2}}\right)^{-2}
$$

where we have used the normalized Killing vector $\zeta^{a}=(\partial / \partial t)^{a} / \Xi$ instead of $(\partial / \partial t)^{a}$ in Eq. (16)

so that the conserved quantities at infinity generate the $S O(3,2)$ algebra, in agreement with the conventions of Ref. [31]. The mass $\mathcal{M}$ as $r$ goes to infinity then becomes

$$
\mathcal{M}_{\infty}=\frac{m}{\Xi^{2}}
$$

which differs from that of eq.(21) by this normalization factor. The $A$ and $M$-dependence of $\mathcal{M}$ are illustrated in Figs. 18 and 19 .

Adopting the same stance as for the Kerr black hole, we express the energy as a function of $R, J$ and $S$. Noting that the entropy is $S=\frac{\pi}{\Xi}\left(r_{+}^{2}+a^{2}\right)$, the temperature can be written as

$$
T=\left(\frac{\partial E}{\partial S}\right)_{J, R}=\left(\frac{\partial E}{\partial M_{\infty}}\right)_{J, R}\left(\frac{\partial S}{\partial M_{\infty}}\right)_{J, R}^{-1}=\frac{\kappa_{+}}{2 \pi R}\left(\frac{\partial E}{\partial M_{\infty}}\right)_{R, J}
$$

where $\kappa_{+}$is the surface gravity at the event horizon

$$
\kappa_{+}=\frac{3 r_{+}^{4}+r_{+}^{2}\left(a^{2}+l^{2}\right)-a^{2} l^{2}}{2 l^{2} r_{+}\left(r_{+}^{2}+a^{2}\right)}
$$

and $M_{\infty}=\mathcal{M}_{\infty} / R$.

As for the Kerr case, the temperature must be computed numerically. We find (fig. 20) that for small values of $M$ the temperature is a weakly decreasing function of $A$, and (similar to the 


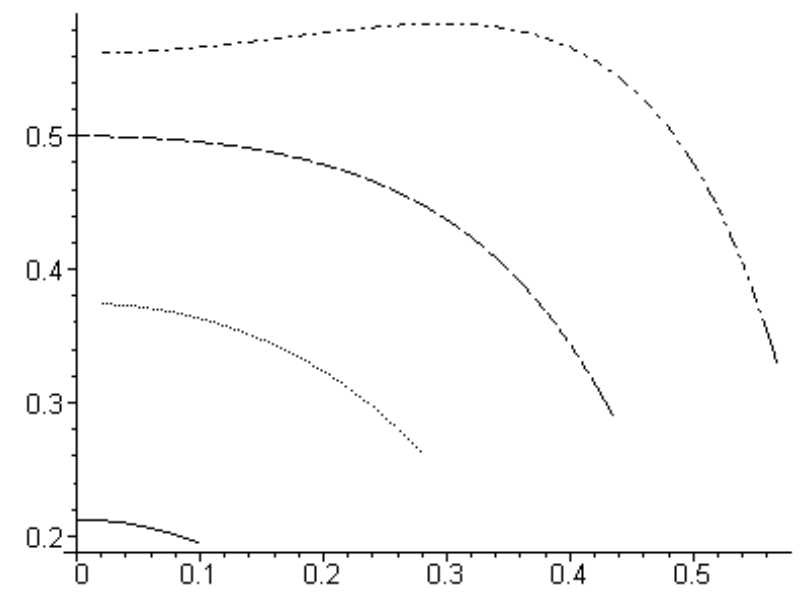

Figure 18: $\mathcal{M} / R$ versus $A$ for $L=1, M=0.1$ (solid), 0.3 (dotted), 0.5 (dashed), 0.7 (dot-dashed). 


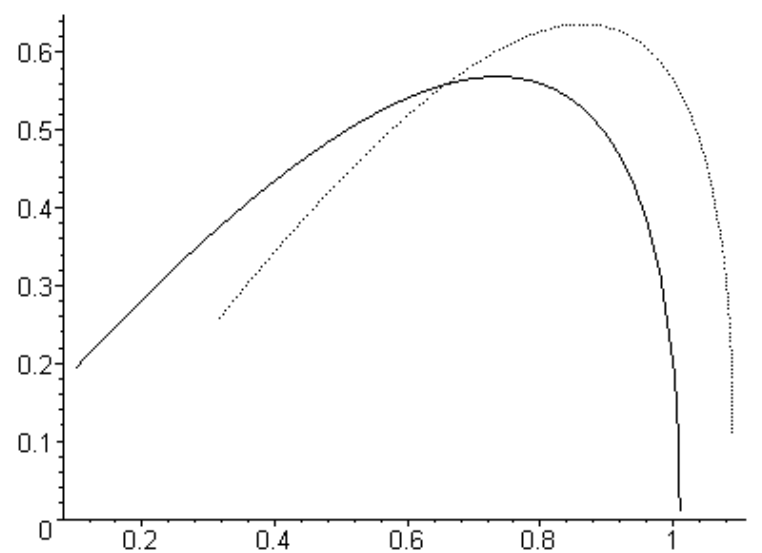

Figure 19: $\mathcal{M} / R$ versus $M$ for $L=1, A=0.3$ (solid), 0.5 (dotted). 


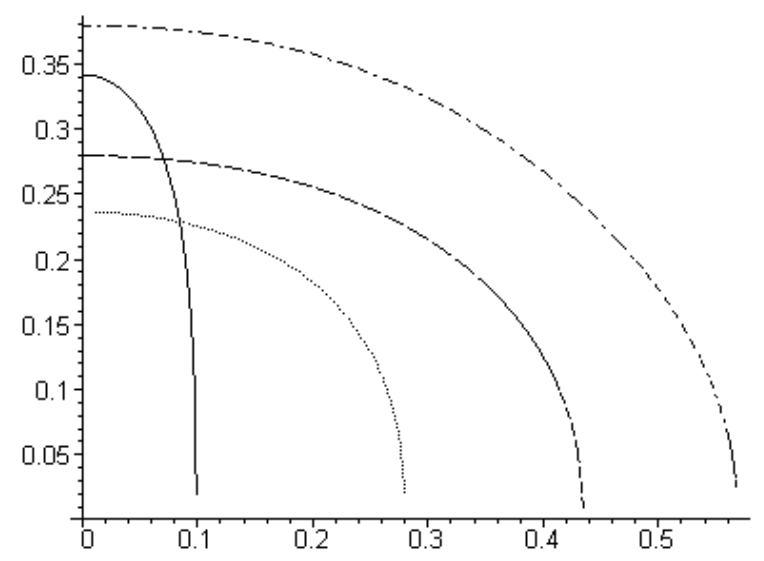

Figure 20: $R T$ versus $A$ for $L=1, M=0.1$ (solid), 0.3 (dotted), 0.5 (dashed), 0.7 (dot-dashed).

Kerr case) it decreases rapidly as $A$ approaches its maximum allowed value. As $M$ increases, the $A$-dependence of $T$ becomes more relevant. The $L$-dependence of the temperature can be seen from fig. 21. Note that the value of $T$ due to the counterterm is very small compared to its value due to the boundary term. In contradistinction to the energy, we find that $T$ is an initially increasing function of $L$ for small $M$. For large values of $L \quad(L>100)$ the change in $T$ due to the boundary term is negligible and $T$ increases as $L$ increases. The $M$-dependence of $T$ is plotted in 22 .

In figure 23 we plot the $M$-dependence of $\left(\frac{\partial T}{\partial M}\right)_{J, R}$. Again this figure is in consistent with that of Kerr case, and shows that for $A=0.05, T(M, J, R)$ has a maximum at $A \simeq 0.078$ and a minimum at $A \simeq 0.25$. We find for small values of $A$ a single stable phase consisting of a small black hole. As the temperature increases, a second stable phase emerges consisting of a large black hole, along with an intermediate-mass unstable phase. This latter phase coalesces with the small black hole at an even larger temperature, beyond which there exists only the single stable large black hole phase. As $A$ increases the small and unstable phases are present for continually smaller 


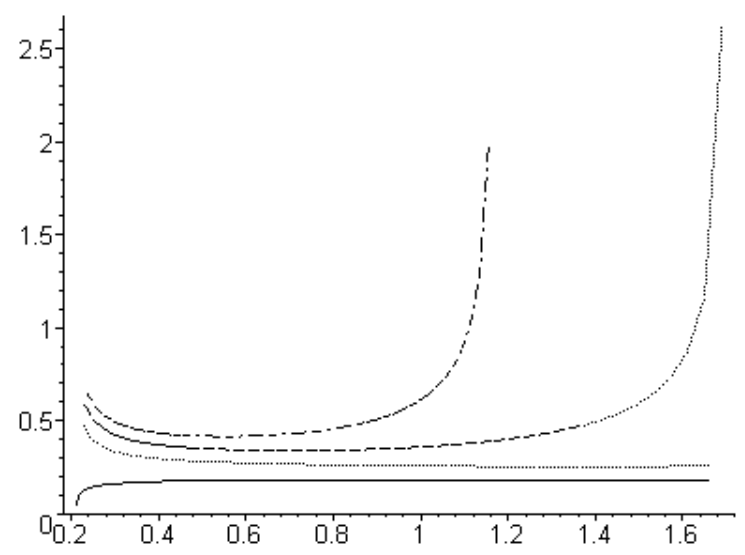

Figure 21: $R T$ versus $L$ for $A=0.2, M=0.3$ (solid), 0.5 (dotted), 0.7 (dashed), 0.9 (dot-dashed). 


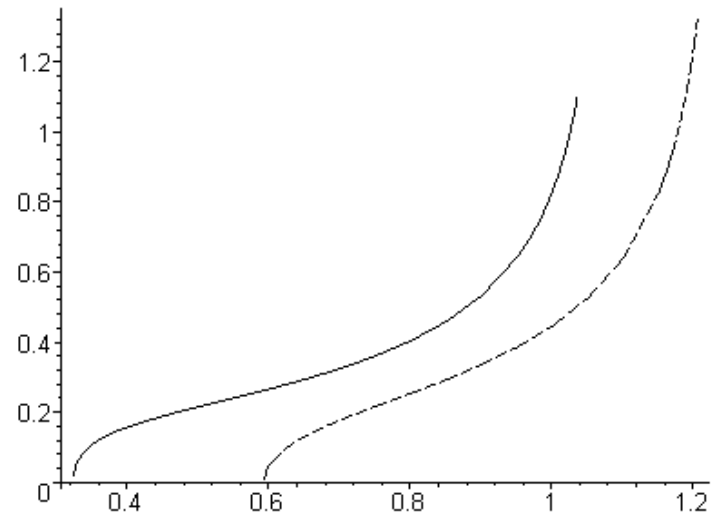

Figure 22: $R T$ versus $M$ for $L=1, A=0.3$ (solid), 0.5 (dashed). 


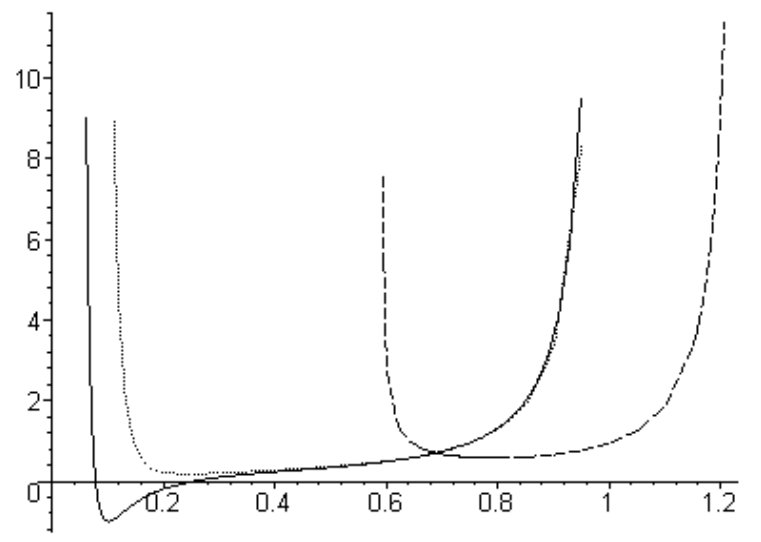

Figure 23: $R\left(\partial T / \partial M_{\infty}\right)_{J, R}$ versus $M$ for $L=1, A=0.05$ (solid), 0.1 (dashed), 0.5 (dashed). 


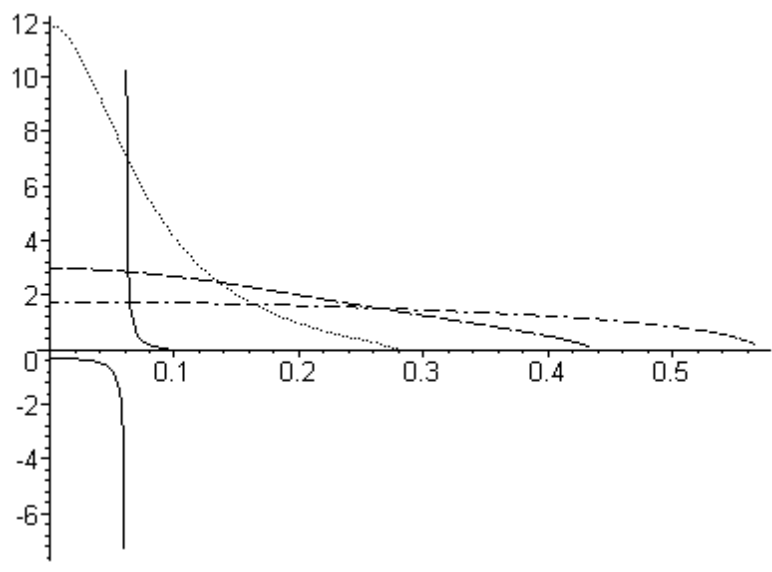

Figure 24: $R^{-2} C_{R}$ versus $A$ for $L=1, M=0.1$ (solid), 0.3 (dotted), 0.5 (dashed), 0.7 (dotdashed).

temperature ranges. They eventually vanish for $A$ sufficiently large, leaving only the single large black hole phase. The heat capacity at constant surface area $4 \pi R^{2}$ can be evaluated numerically, and the results, plotted as functions of $A$ and $M$, are shown in Figs. 24 and 25.

The stability analysis for these black holes is in qualitative agreement with that of Caldarelli et. al. [22], who recently performed a thorough analysis of the thermodynamic properties of Kerr-Newman AdS black holes in the $R \rightarrow \infty$ limit. However they computed a generalization of the Smarr formula for the AdS case in terms of $\mathcal{M}_{\infty}$, whereas we are considering the quasilocal energy $E$ in (46) as a function of $R, S$, and $J$. As a consequence the temperature they derive does not redshift to zero in the large- $R$ limit, unlike the expression in (56) which does, although the explicit form differs from that of the Tolman factor due to the quasilocal surface not being an isotherm when $J \neq 0$.

The angular velocity can be written as:

$$
\Omega=\left(\frac{\partial E}{\partial J}\right)_{S, R}=\left(\frac{\partial E}{\partial M_{\infty}}\right)_{S, R}\left(\frac{\partial M_{\infty}}{\partial J}\right)_{S, R}=\Omega_{E} \frac{1}{R}\left(\frac{\partial E}{\partial M_{\infty}}\right)_{S, R}
$$




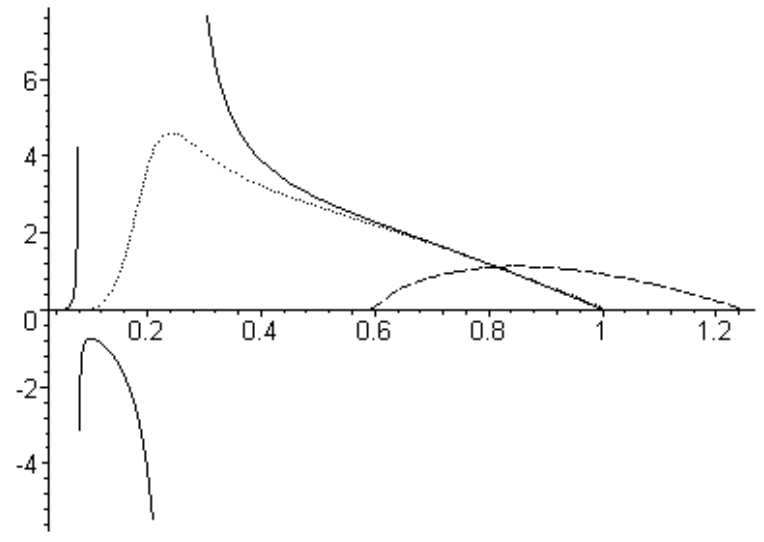

Figure 25: $R^{-2} C_{R}$ versus $M$ for $L=1, A=0.05$ (solid), 0.1 (dotted), 0.5 (dashed). 


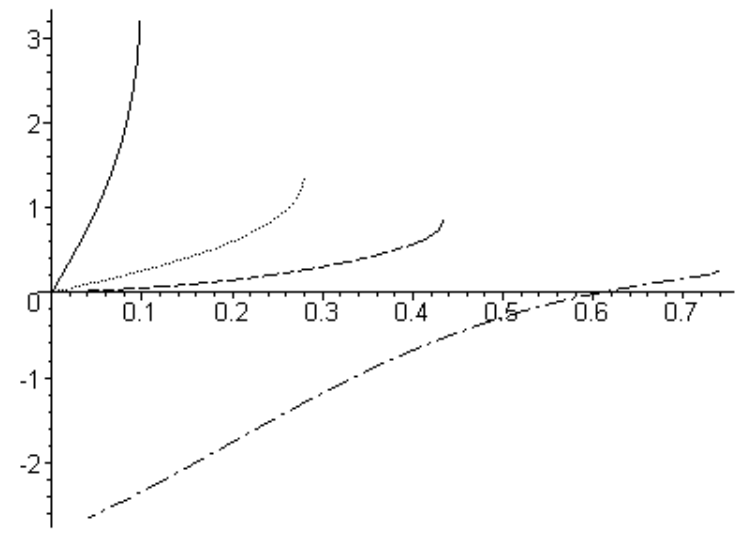

Figure 26: $R \Omega$ versus $A$ for $L=1, M=0.1$ (solid), .3 (dotted), .5 (dashed), 1.0 (dot-dashed).

where $\Omega_{E}$ is the angular velocity of the Einstein universe:

$$
\Omega_{E}=\frac{a\left(1+\frac{r_{+}^{2}}{l^{2}}\right)}{r_{+}^{2}+a^{2}}=\Omega_{H}-\Omega_{\infty}
$$

with $\Omega_{H}=a\left(1-a^{2} / L^{2}\right) /\left(r_{+}^{2}+a^{2}\right)$ the angular velocity of the hole at event horizon, and $\Omega_{\infty}=$ $-a / l^{2}$ is the angular velocity at infinity. As $R \rightarrow \infty,\left(\partial E / \partial M_{\infty}\right)_{S, R} \rightarrow 1$, and the results of Ref. [22] are recovered. The $A$ dependence of the angular velocity can be seen from 26. As in the Kerr case, we find that $\left(\partial E / \partial M_{\infty}\right)_{S, R}$ is not positive once $M$ is sufficiently large. Again, although the numerical computation required to extrapolate the curve for $M=1$ is more intense than the computational power we have available, we have checked explicitly that $\Omega$ vanishes for small $A$ in this case.

\section{$5 \quad 2+1$ BTZ Black Hole}

We can analyze the $(2+1)$-dimensional axially symmetric black hole solution of Einstein equation with a negative cosmological constant $\Lambda=-1 / l^{2}$ obtained by Banados et.al. using methods 
similar to those given above. Written in stationary coordinates the BTZ metric is [32]

$$
d s^{2}=-N^{2}(r) d t^{2}+f^{-2}(r) d r^{2}+r^{2}\left[V^{\phi}(r) d t+d \phi\right]^{2},
$$

with

$$
N^{2}(r)=f^{2}(r)=-m+\left(\frac{r}{l}\right)^{2}+\left(\frac{j}{2 r}\right)^{2}, \quad V^{\phi}(r)=-\frac{j}{2 r^{2}} .
$$

The action becomes

$$
I=-\frac{1}{8 \pi}\left\{\int_{\mathcal{M}} d^{4} x \sqrt{-\gamma}(\mathcal{R}-2 \Lambda)+2 \int_{\partial \mathcal{M}} d^{3} x \sqrt{-\gamma} \Theta-\frac{2}{l} \int_{\partial \mathcal{M}} d^{3} x \sqrt{-\gamma}\right\},
$$

where we drop the counterterm corresponding to the induced Ricci scalar since it makes no contribution at infinity in $(2+1)$ dimensions. The analogue of eq. (45) is

$$
Q^{i j}=\frac{\sqrt{-\gamma}}{2 \pi}\left(-\frac{1}{l} \gamma^{i j}\right)
$$

from which it is easy to show that the energy is

$$
E=2\left(\frac{r}{l}-\sqrt{-m+\frac{r^{2}}{l^{2}}+\frac{j^{2}}{r^{2}}}\right) .
$$

This expression is identical to the one given in Ref. [11, in which the reference spacetime was taken to be that of a zero-mass black hole. We see here that the counterterm prescription at arbitrary $r$ is equivalent to this choice of spacetime background, and so all of the results of Ref. [11] will hold.

\section{Closing Remarks}

Our investigations of the boundary-induced counterterm prescription (田) at finite distances has allowed us to obtain a number of interesting results, which we recapitulate here.

We have been able to compute for the first time the energy, conserved mass, and angular momentum quasilocally for arbitrary values of the parameters of the Kerr and Kerr-AdS solutions, apart from the mild reality restrictions (33) and (52). These quasilocal quantities are intrinsically calculable at fixed $r=R$ without any reference to a background spacetime, a marked improvement over the methods given in Ref. [13], which were necessarily restricted to small values of the rotation parameter. We recovered these results, and were further able to show that the conjecture that $E\left(r_{+}\right)=2 M_{i}$ (where $M_{i}$ is the irreducible mass of the Kerr black hole) was not correct, but instead was valid only for small $A$. We also found that quasilocal angular momentum equals its value at infinity at any fixed value $r=R$. The remarkable result holds because the counterterms integrate to zero, and not because they identically vanish.

We found that the entropy $S$ and angular momentum $J$ were not given by surface integrals over quasilocal boundary data, but rather were constants dependent on the parameters $a, m$ (and 
l) of the black hole. We therefore chose to regard the interior of the quasilocal surface as a thermodynamic system whose energy $E$ was a function of $S$ and $J$. A stability analysis yielded results that were in qualitative agreement with previous investigations carried out for either the non-rotating case [11], or at infinity [21, 22]. This stability analysis is, of course, local, and phase transitions to other spacetimes of lower free energy might exist. For the BTZ black hole the boundary countermterm turned out to be formally identical to a contribution from a reference spacetime of zero mass [1], and so the thermodynamics was identical in the two cases. It would be interesting to repeat these investigations for a different choice of quasilocal boundary, such as an isotherm.

The counterterm (4) reduces to the two cases given in eqs. (22) and (43) the large- $l$ and $\mathcal{O}(l)$ limits respectively. These two regimes are quite distinct except when the boundary curvature scalar $\mathcal{R}(\gamma) \sim 1 / l$, at which point one might expect that other boundary curvature invariants besides the minimal number we have considered here would be relevant. However we have not found it necessary to include such terms.

Indeed, boundary counterterms evidently have a robust degree of applicability, going well beyond that originally proposed from their origins in the AdS/CFT correspondence conjecture. In some ways this is not too surprising, since one need have no a-priori knowledge of the conjecture to introduce such terms. However we have found the somewhat unexpected result that inclusion of the minimal number of intrinsic boundary terms needed to make quantities well defined at infinity is sufficient to compute the thermodynamics of both asymptotically flat and asymptotically AdS black holes for virtually all possible values of the rotation parameter and cosmological constant that leave the quasilocal boundary well defined. This goes well beyond what is possible with background subtraction methods, and perhaps suggests that a deeper physical meaning be ascribed to such terms.

\section{Acknowledgments}

M.H.D. is grateful for the hospitality of the Department. of Physics of Waterloo University, where this work was performed, and for partial support from the Research council of Shiraz University of Iran. We would like to thank I. Booth for interesting correspondence. This work was supported by the Natural Sciences and Engineering Research Council of Canada.

\section{References}

[1] J.D. Beckenstein, Phys. Rev. D7, 2333 (1973).

[2] S.W. Hawking, Nature 248, 30 (1974) ; Comm. Math. Phys. 43, 199 (1975)

[3] G.W. Gibbons and S.W. Hawking, Comm. Math. Phys. 66, 291 (1979).

[4] S.W. Hawking and C. Hunter, Phys.Rev.D 59, 044025 (1999).

[5] R.B. Mann, Phys.Rev. D 60, 104047 (1999).

[6] R. Emparan, C.V. Johnson and R. Meyers, Phys.Rev. D 60, 104001 (1999). 
[7] S.W. Hawking, C. Hunter and D. Page, Phys.Rev. D 59, 044033 (1999).

[8] R.B. Mann, Phys.Rev. D 61, 084013 (2000).

[9] L. Fatibene, M. Ferraris, M. Francaviglia, M. Raiteri, gr-qc/0003019.

[10] J.D. Brown and J.W. York, Phys. Rev. D 47, 1407 (1993).

[11] J.D. Brown, J. Creighton and R.B. Mann, Phys. Rev. D 50, 6394 (1994).

[12] K.C.K. Chan, J.D.E. Creighton, and R.B. Mann Phys. Rev. D 543892 (1996).

[13] E. Martinez, Phys. Rev. D 504920 (1994).

[14] M. Hennigson and K. Skenderis, JHEP 07, 023 (1998)

[15] S.Y. Hyun, W.T. Kim and J. Lee, Phys. Rev. D 59084020 (1999)

[16] V. Balasubramanian and P. Kraus, Commun.Math.Phys. 208413 (1999) (hepth/9902121).

[17] S. N. Solodukhin, Phys.Rev. D 62: 044016 (2000) (hep-th/9909197).

[18] P. Kraus, F. Larsen and R. Siebelink, Nucl. Phys. B 563259 (1999).

[19] A. M. Awad and C. V. Johnson, Phys.Rev. D 61084025 (2000).

[20] A. DeBenedictus and K.Vishwanathan, (hep-th/9911060).

[21] P. C. W. Davies, Proc. R. Soc. London A., 353, 499 (1977).

[22] M. Caldarelli, G. Cognola and D. Klemm, Class.Quant.Grav. 17 (2000) 399 hepth/9908022).

[23] I.S. Booth and R.B. Mann, Phys.Rev. D 59064021 (1999) (gr-qc/9810009)

[24] J.D.E. Creighton, "Gravitational Calorimetry" (Ph.D. Thesis) gr-qc/9610038.

[25] I.S. Booth and J.D.E. Creighton, Phys.Rev. D 62067503 (2000) gr-qc/0003038)

[26] S.R. Lau, Phys.Rev. D 60104034 (1999) (gr-qc/9903038).

[27] A. Ashtekar and S. Das, Class.Quant. Grav. 17 L17 (2000) (hep-th/9911230)

[28] S. Das and R.B. Mann, J.H.E.P. 08033 (2000) (hep-th/0008028)

[29] D. Christodolou and R. Ruffini, Phys. Rev. D4 3554 (1971).

[30] J.D. Brown, E. Martinez and J.W. York, Proc. VIth Florida Workshop on Nonlinear Astronomy, 226 (eds. S. Detweiler and J. Ipser), Ann. N.Y. Acad. Sci. (1990). 
[31] V.A. Kostelecky and M. J. Perry, Phys.Lett. B 371191 (1996) (hep-th/9512222)

[32] M. Bañados, C. Teitelboim, and J. Zanelli, Phys. Rev. Lett. 691849 (1992); M. Bañados, M. Henneaux, C. Teitelboim, and J. Zanelli, Phys. Rev. D 481506 (1993); 